\title{
Unreported and Overlooked: A Post Hoc Analysis of COPD Symptom-Related Attacks from the RISE Study
}

This article was published in the following Dove Press journal: International Journal of Chronic Obstructive Pulmonary Disease

\author{
Gary T Ferguson' \\ Tor Skärby $\mathbb{D}^{2}$ \\ Lars H Nordenmark (iD $)^{2}$ \\ Rosa Lamarca ${ }^{3}$ \\ Audrone Aksomaityte ${ }^{4}$ \\ Dan Lythgoe (D) ${ }^{4}$ \\ Ileen Gilbert (iD ${ }^{5}$ \\ Frank Trudo 5 \\ 'Department of Medicine, Pulmonary \\ Research Institute of Southeast Michigan, \\ Farmington Hills, MI, USA; \\ ${ }^{2}$ BioPharmaceuticals R\&D, AstraZeneca, \\ Gothenburg, Sweden; ${ }^{3}$ BioPharmaceuticals \\ R\&D, AstraZeneca, Barcelona, Spain; \\ ${ }^{4}$ Statistics Department, Phastar, London, \\ UK; ${ }^{5}$ BioPharmaceuticals Medical - US, \\ AstraZeneca LP, Wilmington, DE, USA
}

Correspondence: Gary T Ferguson MD, Pulmonary Research Institute of Southeast Michigan, 29255 West 10 Mile Road, Suite A, Farmington Hills, MI 48336, USA

Tel +| $248478656 \mid$

Email garytferguson@msn.com
Purpose: Moderate and severe COPD exacerbations are a significant health-care burden, but patients also experience "mild" exacerbations, or COPD symptom-related attacks, which often go unreported. We aimed to define and then determine the incidence of COPD symptom-related attacks and their impact on future risk of moderate/severe exacerbations, health-related quality of life (HRQoL), and lung function. The effect of COPD maintenance therapy on the attack definition was then evaluated by comparing budesonide/formoterol with formoterol alone.

Patients and Methods: This post hoc analysis of the RISE study defined COPD symptomrelated attacks as $\geq 2$ consecutive days of both worsening symptoms and increased daily rescue medication use based upon thresholds of $>2$ and $>4$ short-acting $\beta_{2}$-agonist (SABA) inhalations/day above baseline. The impact of these events on subsequent moderate/severe exacerbation risk was estimated using a time-varying Cox proportional hazards model. The effects of COPD symptom-related attacks on St George's Respiratory Questionnaire (SGRQ) total score and pre-bronchodilator forced expiratory volume in 1 second $\left(\mathrm{FEV}_{1}\right)$ were evaluated as average changes from baseline to first post-attack measurement. Rates of attacks were compared between treatment groups using negative binomial regression models.

Results: COPD symptom-related attacks elevated the risk of subsequent moderate/severe exacerbations at both $>2$ and $>4$ inhalations/day above baseline (HR 1.86 and 2.21, respectively; $\mathrm{p}<0.0001$ ), with a cumulative increase in risk with increasing attacks. HRQoL and lung function were reduced for patients with $\geq 1$ versus no COPD symptom-related attacks at both rescue medication thresholds. There were fewer COPD symptom-related attacks with budesonide/formoterol versus formoterol alone, with no increased risk of pneumonia and lower respiratory tract infections.

Conclusion: COPD symptom-related attacks are common and typically unreported. Importantly, these attacks can account for considerable morbidity and should not be regarded as "mild". Detection of such exacerbations may be valuable in identifying patients at greater risk and guiding preventive therapeutic interventions.

Keywords: COPD, exacerbation, symptoms, rescue inhaler, mild COPD exacerbations

\section{Introduction}

Moderate and severe chronic obstructive pulmonary disease (COPD) exacerbations - as defined by health-care resource utilization (HCRU) intensity, including treatment with oral corticosteroids (OCS), antibiotics and/or hospitalization - are a significant burden on patients and health-care systems. ${ }^{1}$ In addition, exacerbations can accelerate lung function decline associated with COPD, reduce patients' 
physical activity, worsen their health-related quality of life (HRQoL), and increase the risk of death. ${ }^{2,3}$ In the United States, acute COPD exacerbations account for $3.4 \%$ of all hospitalizations, ${ }^{4}$ with yearly total costs for COPD estimated at $\$ 50$ billion, of which $\$ 30$ billion was due to direct health-care expenditure. ${ }^{5}$

Apart from moderate and severe exacerbations, patients with COPD often experience periods of worsening symptoms that result in them intensifying therapy with short-acting bronchodilators. These events are associated with symptoms typical of COPD exacerbations, including increasing dyspnea, cough and sputum changes. Importantly, these events are rarely identified in clinical practice, thus not treated with systemic corticosteroids or antibiotics. Indeed, these patients usually do not present to their health-care professionals with their complaints, whether by choice or due to lack of awareness. ${ }^{6,7}$

Some studies have reported findings in patients with symptoms associated with COPD worsenings that are not treated with OCS/antibiotics and/or hospitalization. They suggest up to half of these "events", as identified by analyses of daily symptom diaries, are never reported by patients. ${ }^{7,8}$ A study evaluating the frequency and impact of exacerbations identified by HCRU versus those identified using a patient-reported daily diary with the EXAcerbations of Chronic pulmonary disease Tool $\left(\right.$ EXACT $^{\circledR}$ ) determined that over two-thirds of the diarydefined events went unreported. The unreported events had as great an impact on medium-term health status as the reported events. ${ }^{9}$ Importantly, over one-third of patients reporting their symptoms as only mild-to-moderate in severity also stated they were too breathless to leave home. $^{10}$

The Global Initiative for Chronic Obstructive Lung Disease (GOLD) report describes such acute worsenings of respiratory symptoms as "mild" exacerbations, ${ }^{1}$ despite the fact they may have a significant impact on health status. ${ }^{6,711}$ Unfortunately, for most patients, daily use of an assessment tool to identify "mild" exacerbations is not feasible.

If the rate of "mild" exacerbations, or "COPD symptom-related attacks", could be easily quantified, and if their impact was known, these data could potentially be used as a simple clinical tool and predictor for future moderate/severe exacerbations, facilitating more appropriate preventative action. ${ }^{6}$ Having a quantifiable definition of such events could also allow the effectiveness of preventative therapeutic interventions to be determined, and indicate for whom the benefit/risk profiles of such interventions may be favorable.

More information on "mild" COPD exacerbations is essential for better understanding of the need for care in patients with COPD. To our knowledge, reliever inhaler usage linked to worsening symptoms has not been previously assessed in COPD patients to understand the potential impact of these events on patient health status and risk of moderate-to-severe COPD exacerbations.

The RISE study has previously shown that budesonide/ formoterol pressurized metered dose inhaler (pMDI) 320/9 $\mu \mathrm{g}$ twice daily (BID) effectively reduced moderate and severe exacerbations versus formoterol dry powder inhaler (DPI) $9 \mu \mathrm{g}$ BID alone in COPD patients with moderate-tovery-severe airflow limitation and a history of moderate or severe exacerbations. ${ }^{12}$ Patients enrolled in the RISE study completed a daily electronic diary (eDiary), providing daily data on symptoms and rescue medication usage, and allowing an opportunity to analyze how changes in these parameters outside of moderate and severe COPD exacerbations relate to patient morbidity.

In this post hoc analysis of RISE study data, we identify and assess the impact of varying levels of rescue medication usage to develop a clinically meaningful definition of "COPD symptom-related attacks" and quantify their incidence. We then assess the importance of these attacks in terms of risk of future moderate/severe exacerbations and the impact on HRQoL and lung function. Finally, we assess whether therapy with budesonide/formoterol versus formoterol alone can reduce the frequency of, and outcomes associated with, COPD symptom-related attacks, and whether this addition of an inhaled corticosteroid (ICS) to a long-acting $\beta_{2}$-agonist (LABA) is associated with an increased incidence of lower respiratory tract infections (LRTI) and pneumonia.

\section{Patients and Methods}

The RISE study (ClinicalTrials.gov identifier: NCT02157935) methodology has been described previously. ${ }^{12}$ Briefly, patients $(\mathrm{N}=1,219)$ aged $\geq 40$ years with moderate-to-very-severe COPD (per lung function) and a history of $\geq 1$ moderate or severe COPD exacerbation in the previous year were randomized to budesonide/formoterol pMDI 320/9 $\mu \mathrm{g}$ BID ( $\mathrm{n}=606)$ or formoterol DPI 9 $\mu \mathrm{g}$ BID ( $\mathrm{n}=613$ ) for 26 weeks. Patients were ineligible for the study if their usual maintenance or rescue therapy had changed due to worsening symptoms within 14 days of enrollment and up to randomization. Patients were also 
excluded if they experienced moderate or severe COPD exacerbations that had not resolved within 14 days of enrollment or that occurred between enrollment and runin.

In this post hoc analysis, COPD symptom-related attacks were defined as $\geq 2$ consecutive days of both worsening symptoms and increased daily rescue medication use (either $>2$ or $>4$ inhalations of SABA per day above baseline). Modified Anthonisen criteria $^{13}$ were used for worsening of symptoms, with either a worsening of $\geq 2$ major symptoms (dyspnea, sputum volume, or sputum color/purulence) or of 1 major symptom and $\geq 1$ minor symptom (sore throat, colds, fever, cough, or wheeze). The decision to use increased daily rescue medication use - and the thresholds of $>2$ and $>4$ SABA inhalations/ day above baseline - as well as worsening symptoms was an effort to distinguish COPD symptom-related attacks from the normal day-to-day variability in COPD symptoms. Details of how symptoms and rescue medication usage were recorded in the daily electronic diary are provided in the Supplementary Appendix.

Baseline rescue medication use was defined as the mean of the values recorded morning and evening during the 10 days prior to randomization (when all patients were receiving budesonide/formoterol). In an attempt to ensure good compliance with the eDiary, entry into randomization required a minimum of 13 valid values during this time, including the evening assessment 10 days prior to randomization and the morning assessment on the day of randomization. To account for missing data, the mean baseline rescue medication use was calculated for the actual number of days on which evaluable diary entries were recorded. eDiary compliance during the 10 days prior to randomization was assessed to determine baseline rescue medication use. The effect of COPD symptom-related attacks on risk of subsequent future moderate/severe exacerbations, HRQoL and lung function was assessed using thresholds of $>2$ SABA inhalations/day above baseline and $>4$ SABA inhalations/day above baseline for the definition of increased rescue medication use (ie $>1$ extra dose or $>2$ extra doses above baseline, based on two inhalations per dose).

Cox proportional hazards models were used to assess the association of attacks to subsequent moderate/severe exacerbations. A first COPD symptom-related attack was included as a time-varying factor in a multivariable model, additionally adjusted for randomized treatment, country, and number of exacerbations in the prior year $(\leq 1,2$,
$>2$ ). In an additional analysis, separate time-varying factors were fitted for the first and second (or subsequent) COPD symptom-related attacks.

HRQoL and lung function were evaluated via change from baseline to first St George's Respiratory Questionnaire (SGRQ) total score and pre-bronchodilator forced expiratory volume in 1 second $\left(\mathrm{FEV}_{1}\right)$ recorded after the onset of COPD symptom-related attack, using descriptive statistics. SGRQ and $\mathrm{FEV}_{1}$ were measured at baseline (randomization) and weeks $4,8,17$, and 26. The SGRQ was completed by patients during each clinic visit and was performed before all other assessments. Prebronchodilator lung function testing was also conducted at each clinic visit. Patients were instructed not to use study medication within 12 hours of spirometry, and not to use rescue medication within 6 hours. When attacks were absent over the course of the study, SGRQ and $\mathrm{FEV}_{1}$ assessments were averaged over 26 weeks. When these attacks occurred during the study, the first $\mathrm{SGRQ} / \mathrm{FEV}_{1}$ assessment after the onset of the attack was used, or if a patient experienced more than one attack, the mean was used.

To support the assessment of the impact of COPD symptom-related attacks, negative binomial regression models were used to compare rates of attacks between treatment groups. Attacks were considered as part of a moderate/severe exacerbation if they occurred $\leq 7$ days before/after the exacerbation and were not counted as COPD symptom-related attacks. COPD symptom-related attacks occurring $\leq 7$ days apart were counted as one. Covariates included treatment group, baseline rescue medication use, country, and exacerbations in the prior year $(\leq 1,2,>2)$. Other outcome measures compared between treatment groups were attack duration, absolute and change from baseline SABA use, and incidence of LRTI and pneumonia events, which are risks that have been associated with ICS use. $^{14}$ LRTI and protocol-defined pneumonia events were recorded as adverse events (AEs) or serious adverse events (SAEs) as part of the overall RISE study safety evaluation.

The RISE study was performed in accordance with the Declaration of Helsinki and Good Clinical Practice. Written informed consent was provided by all patients before participation in the study. The final study protocol was approved by an independent ethics committee. Details of the ethics committee for each study center are provided in the Supplementary Appendix. 


\section{Results}

\section{Patients}

Patient demographics and baseline characteristics are given in Table 1. On-treatment eDiary compliance was $\geq 94 \%$ in both treatment groups. Mean eDiary compliance over the last 10 days prior to randomization was $>97 \%$.

\section{Rescue Medication Use}

Mean baseline rescue medication use for all patients was 1.46 inhalations/day. Mean rescue inhalations per event day during COPD symptom-related attacks were 7.18 at the $>2$ SABA inhalations/day above baseline threshold and 10.25 at the $>4$ inhalations/day above baseline threshold. Comparatively, mean rescue medication use during a moderate/severe exacerbation was 2.49 inhalations/event day above baseline threshold.

The mean event duration was 5.23 days at the $>2$ inhalations/day threshold, 6.23 days at the $>4$ inhalations/day threshold. The mean event duration for moderate/severe exacerbations was 9.93 days, although the definition of duration was different from that used for COPD symptom-related attacks (Supplementary appendix).

\section{COPD Symptom-Related Attacks}

In total, $16 \%$ of evaluable patients had COPD symptomrelated attacks at the $>2$ inhalations/day threshold. For the $>4$ inhalations/day threshold, $6 \%$ of patients had COPD symptom-related attacks. Comparatively, $31 \%$ of patients experienced moderate/severe exacerbations.

Therefore, for every COPD symptom-related attack at the $>2$ inhalations/day threshold there were 1.72 moderate/ severe exacerbations, and for every COPD symptomrelated attack at the $>4$ inhalations/day threshold there were 4.81 moderate/severe exacerbations. COPD symptom-related attacks accounted for $37 \%$ of all ("mild", moderate and severe) exacerbation events at the $>2$ inhalations/day threshold and $17 \%$ of all exacerbation events at the $>4$ inhalations/day threshold.

\section{Development of Moderate/Severe Exacerbations}

Experiencing any $(\geq 1)$ COPD symptom-related attack elevated the risk of a subsequent moderate/severe exacerbation for both the $>2$ inhalations/day threshold (hazard ratio [HR] 1.86; 95\% confidence interval [CI]: 1.39, 2.49; $\mathrm{p}<0.0001$ ) and the $>4$ inhalations/day threshold (HR 2.21; 95\% CI: 1.48, 3.28; $<<0.0001$ ) (Figure 1A).

Table I Patient Demographics and Baseline Characteristics

\begin{tabular}{|c|c|c|}
\hline Characteristic & BUD/FORM $(n=606)$ & FORM $(n=6 \mid 3)$ \\
\hline Age, years, mean (range) & $63.1(40-87)$ & $63.9(4 \mid-87)$ \\
\hline Men, n (\%) & $355(58.6)$ & $343(56.0)$ \\
\hline \multicolumn{3}{|l|}{ Previous disease-related treatments at enrolment, $\mathrm{n}$ (\%) } \\
\hline ICS-containing therapies & $382(63.0)$ & $391(63.8)$ \\
\hline Mono inhaled long-acting bronchodilator therapy & $170(28.1)$ & $197(32.1)$ \\
\hline Dual inhaled long-acting bronchodilator therapy & $14(2.3)$ & $5(0.8)$ \\
\hline Short-acting bronchodilator & $476(78.5)$ & $481(78.5)$ \\
\hline Systemic corticosteroids & 0 & $\mathrm{I}(0.2)$ \\
\hline LTRA & $14(2.3)$ & $15(2.4)$ \\
\hline PDE-4 inhibitor & $4(0.7)$ & $5(0.8)$ \\
\hline Xanthines & $35(5.8)$ & $35(5.7)$ \\
\hline FEV , pre-bronchodilator, L, mean (SD)* & $1.29(0.48)$ & $1.31(0.50)$ \\
\hline $\mathrm{FEV}_{\mathrm{I}} / \mathrm{FVC}$ post-bronchodilator, \%, mean (SD) $\dagger$ & $0.49(0.11)$ & $0.50(0.11)$ \\
\hline \multicolumn{3}{|c|}{ Rescue medication inhalations at baseline, inhalations/day $\ddagger$} \\
\hline Mean (SD) & $1.52(2.16)$ & $1.46(2.21)$ \\
\hline Median & 0.50 & 0.42 \\
\hline
\end{tabular}

Notes: *Assessed at the randomization visit. †Data were from run-in visit. $¥ B$ BU/FORM ( $n=602)$; FORM ( $n=607)$.

Abbreviations: BUD, budesonide; FEV , forced expiratory volume in I second; FORM, formoterol; FVC, forced vital capacity; ICS, inhaled corticosteroid; LTRA, leukotriene receptor antagonist; PDE-4, phosphodiesterase 4; SD, standard deviation. 


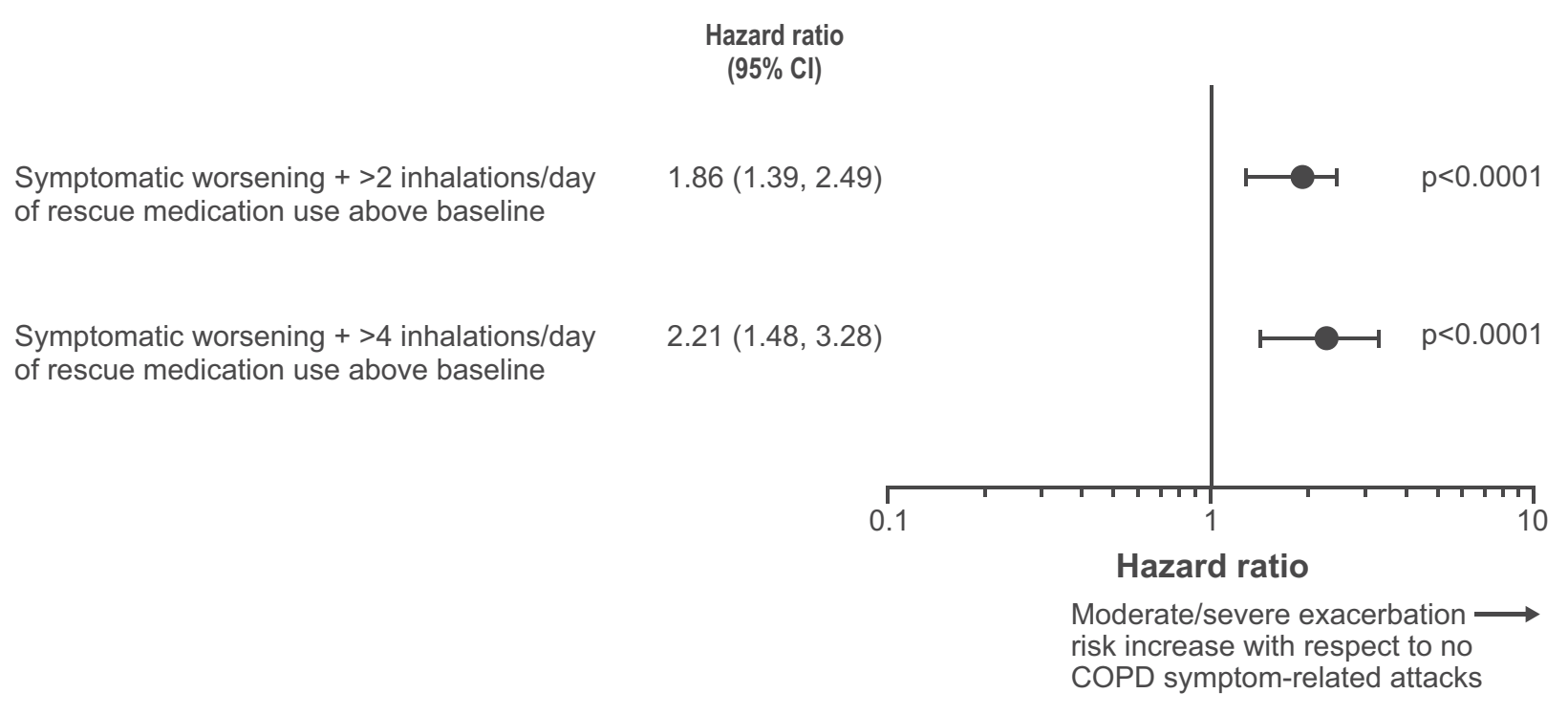

Hazard ratio

$(95 \% \mathrm{Cl})$

Symptomatic worsening $+>2$ inhalations/day

of rescue medication use above baseline

Symptomatic worsening $+>4$ inhalations/day

of rescue medication use above baseline
$2.21(1.48,3.28)$

COPD symptom-related attacks

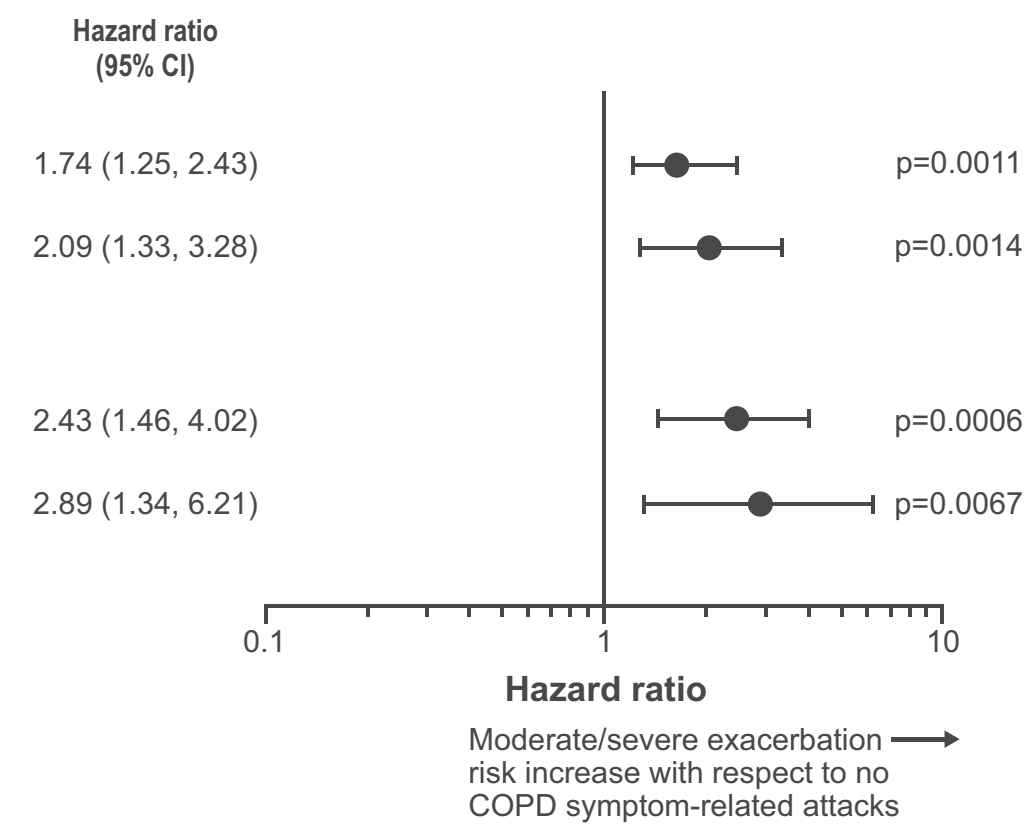

1 vs 0 COPD symptom-related attacks

Symptomatic worsening $+>2$ inhalations/day

of rescue medication use above baseline

Symptomatic worsening $+>4$ inhalations/day

of rescue medication use above baseline

$\geq 2$ vs 0 COPD symptom-related attacks

Symptomatic worsening $+>2$ inhalations/day

of rescue medication use above baseline

Symptomatic worsening $+>4$ inhalations/day

of rescue medication use above baseline

Figure I (A) The effect of experiencing any ( $\geq$ I) COPD symptom-related attack on the risk of a subsequent moderate/severe exacerbation. (B) The effect of experiencing I or $\geq 2$ COPD symptom-related attacks on the risk of a subsequent moderate/severe exacerbation.

Notes: Cox regression with COPD symptom-related attack status as a time-varying covariate was performed to establish the relationship between these events and moderate/severe exacerbations. Covariates of treatment group, country and number of exacerbations in the prior year were included, and a COPD symptom-related attack was categorized as a binary event in (A) and a discrete variable (0, I and >2 events) in (B).

Abbreviation: $\mathrm{Cl}$, confidence interval.

There was a cumulative increase in the risk of experiencing a moderate/severe exacerbation with more attacks. At the $>2$ inhalations/day threshold, for 1 attack versus no attacks, the HR was 1.74 (95\% CI: 1.25, 2.43; $\mathrm{p}=0.0011)$; for $\geq 2$ attacks versus no attacks, the HR was 2.43 (95\% CI: $1.46,4.02 ; \mathrm{p}=0.0006$ ). At the $>4$ inhalations/day threshold, the HR for 1 attack versus no attacks was 2.09
(95\% CI: $1.33,3.28 ; \mathrm{p}=0.0014)$; for $\geq 2$ attacks versus no attacks, the HR was 2.89 (95\% CI: 1.34, 6.21; $\mathrm{p}=0.0067)$ (Figure 1B).

\section{HRQoL}

HRQoL was worse for patients with $\geq 1$ versus no COPD symptom-related attacks (Figure 2). The mean change 


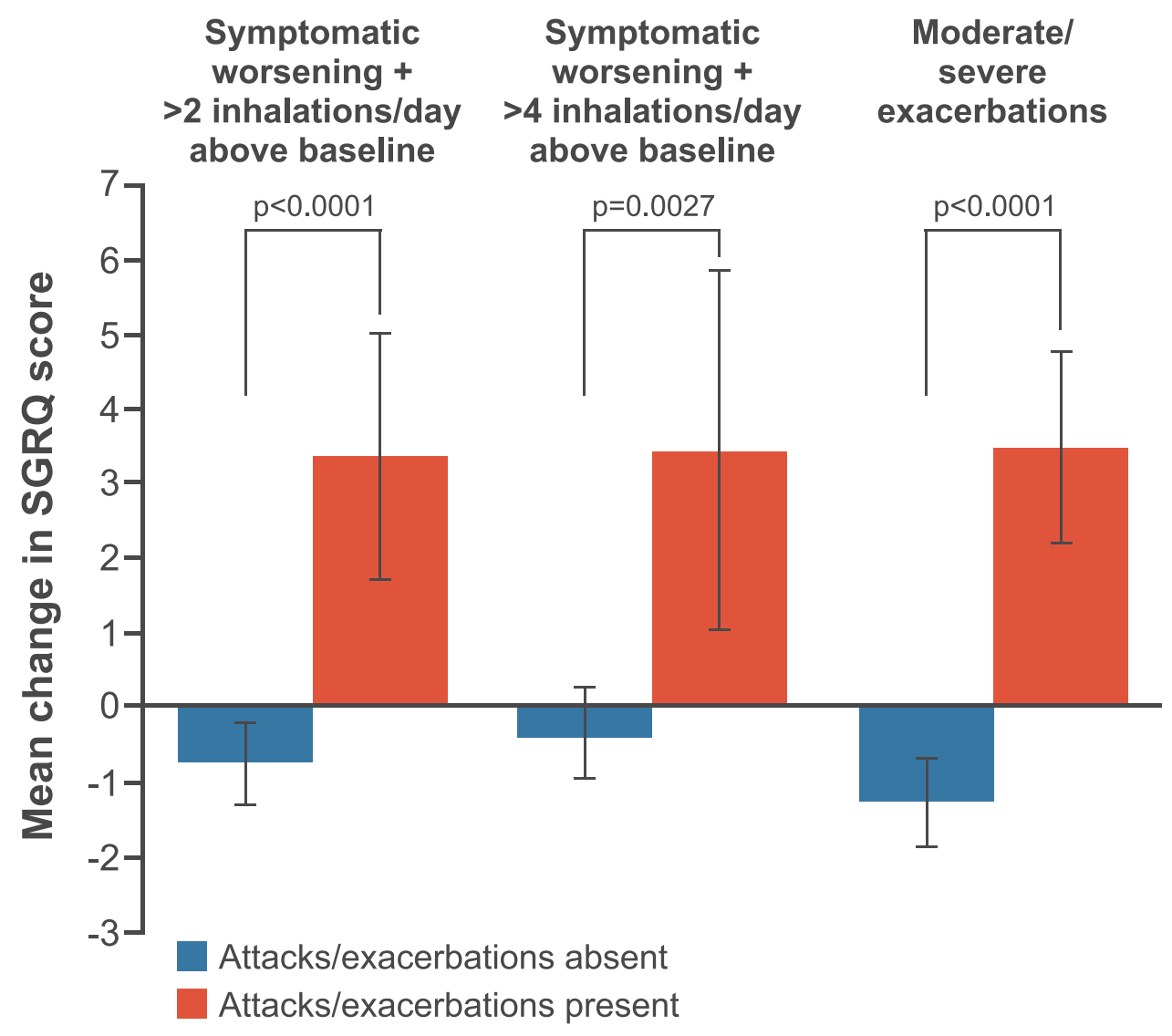

Figure 2 Mean changes in SGRQ total score from baseline.

Notes: Error bars are $95 \% \mathrm{Cl}$. Data are for the change from baseline to first SGRQ total score recorded after the onset of COPD symptom-related attack. Abbreviation: SGRQ, St George's Respiratory Questionnaire.

from baseline in SGRQ total score was -0.76 (95\% CI: $-1.31,-0.20$ ) for patients with no COPD symptom-related attacks and 3.36 (95\% CI: 1.71, 5.00) for patients with $\geq 1$ attack at the $>2$ inhalations/day threshold. At the $>4$ inhalations/day threshold, mean change from baseline in SGRQ total score was $-0.42(95 \% \mathrm{CI}:-0.95,0.11)$ and 3.43 (95\% CI: 1.01, 5.84) for patients with no attacks and $\geq 1$ attack, respectively. This corresponded to a mean difference in change from baseline in SGRQ total score between no attacks and $\geq 1$ attack of 4.11 (95\% CI: 2.38, 5.85) and 3.84 (95\% CI: $1.38,6.31)$ for $>2$ and $>4$ inhalations/day above baseline, respectively.

Similar results were seen for patients with and without moderate/severe exacerbations (Figure 2). The mean change from baseline in SGRQ total score for moderate/severe exacerbations was -1.28 (95\% CI: $-1.87,-0.69)$ for patients with no exacerbations and 3.48 (95\% CI: 2.19, 4.76) for patients with $\geq 1$ exacerbation. The corresponding mean difference for moderate/severe exacerbations was 4.76 (95\% CI: $3.34,6.17$ ) for no exacerbations versus $\geq 1$ exacerbation.

\section{Lung Function}

$\mathrm{FEV}_{1}$ was reduced for patients with $\geq 1$ versus no COPD symptom-related attacks (Figure 3 ). The mean change from baseline in pre-bronchodilator $\mathrm{FEV}_{1}$ at the $>2$ inhalations/day threshold was $0.001 \mathrm{~L}(95 \% \mathrm{CI}:-0.012,0.014)$ for patients with no attacks and $-0.082 \mathrm{~L}(95 \% \mathrm{CI}:-0.118$, -0.046 ) for patients with $\geq 1$ attack. The mean change from baseline at the $>4$ inhalations/day threshold was -0.003 L (95\% CI: $-0.016,0.009)$ and -0.102 L (95\% CI: -0.164 , -0.040 ) for patients with no attacks and $\geq 1$ attack, respectively. The mean difference in change from baseline between patients with no attacks and patients with $\geq 1$ attack was $-0.083 \mathrm{~L}(95 \% \mathrm{CI}:-0.121,-0.045)$ and -0.098 L (95\% CI: $-0.161,-0.036)$ for $>2$ and $>4$ inhalations/day above baseline, respectively.

$\mathrm{FEV}_{1}$ was also reduced for patients with $\geq 1$ versus no moderate/severe exacerbations (Figure 3). The mean change from baseline in pre-bronchodilator $\mathrm{FEV}_{1}$ for moderate/severe exacerbations was $-0.001 \mathrm{~L}(95 \% \mathrm{CI}:-0.015$, 0.013 ) for patients with no exacerbations and -0.016 


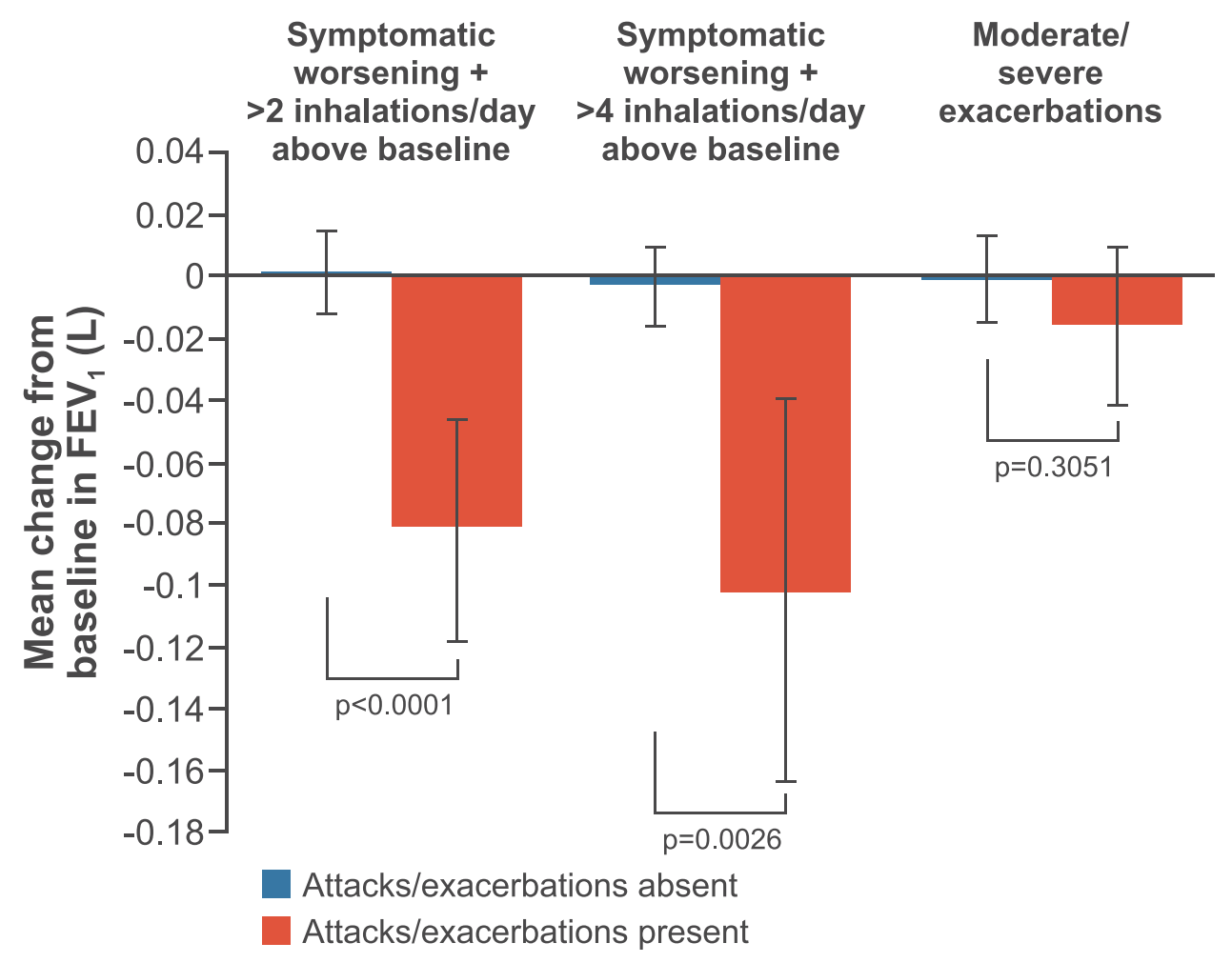

Figure 3 Mean changes in pre-bronchodilator $\mathrm{FEV}_{1}$ from baseline.

Notes: Error bars are $95 \% \mathrm{Cl}$. Data are for the change from baseline to first FEV, assessment recorded after the onset of COPD symptom-related attack. Abbreviation: $\mathrm{FEV}_{1}$, forced expiratory volume in I second.

$$
\begin{aligned}
& \text { Events Patient- } \quad \text { Rate } \quad \text { Rate ratio } \\
& \text { years } \quad(95 \% \mathrm{Cl}) \quad(95 \% \mathrm{Cl})
\end{aligned}
$$

Symptomatic worsening $+>2$ inhalations/day of rescue medication use above baseline

$\begin{array}{lllll}\text { BUD/FORM 320/9 } \mu \mathrm{g} \text { BID } & 111 & 282.0 & 0.27(0.19,0.38) & 0.56(0.41,0.77) \\ \text { FORM } 9 \mu \mathrm{g} \mathrm{BID} & 179 & 271.8 & 0.49(0.36,0.66) & \end{array}$

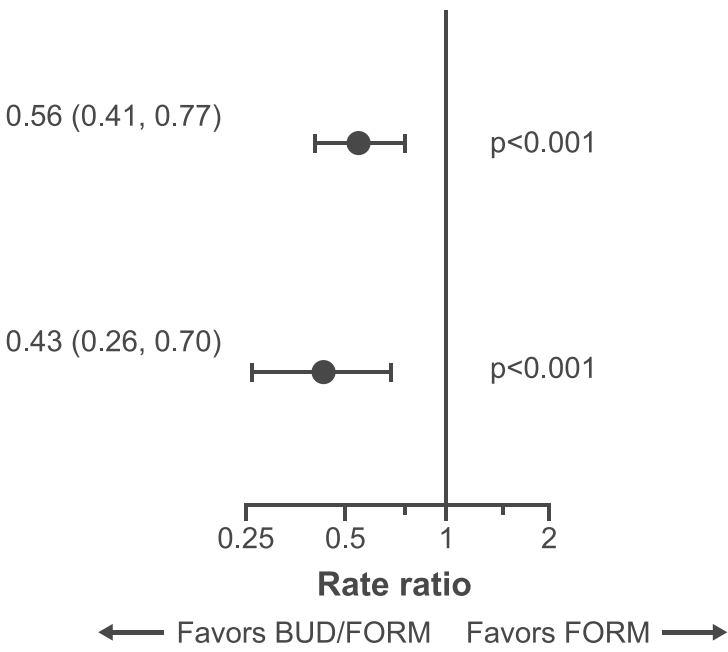

Symptomatic worsening $+>4$ inhalations/day of rescue medication use above baseline

BUD/FORM 320/9 $\mu \mathrm{g}$ BID

33

282.0

$0.05(0.03,0.10)$

$0.43(0.26,0.70)$

FORM $9 \mu \mathrm{g}$ BID

$71 \quad 271.8 \quad 0.12(0.07,0.21)$

Figure 4 COPD symptom-related attack event rates according to use of rescue medication above baseline.

Abbreviations: BID, twice daily; BUD, budesonide; $\mathrm{Cl}$, confidence interval; FORM, formoterol.

L (95\% CI: $-0.040,0.009)$ for patients with $\geq 1$ exacerbation. The mean difference in change from baseline between patients with no exacerbations and patients with $\geq 1$ exacerbation was -0.015 L (95\% CI: $-0.043,0.014)$.
The median time from event onset to lung function measurement was approximately 22 days for the $>2$ and $>4$ inhalations/day thresholds and around 26 days for moderate/severe exacerbations (Table S1). 
Table 2 Characteristics of COPD Symptom-Related Attacks, by Treatment Group

\begin{tabular}{|c|c|c|}
\hline Characteristic & BUD/FORM $(n=606)$ & FORM $(n=6 \mid 3)$ \\
\hline Symptomatic worsening $+>2$ inhalations/day of rescue medication use above baseline & $\mathrm{n}=|\mathrm{I}|$ Events & $n=I 79$ Events \\
\hline \multicolumn{3}{|l|}{ Attack duration (days) } \\
\hline Median (interquartile range) & $2(2-4)$ & $2(2-5)$ \\
\hline Mean (SD) & $4.88(7.75)$ & $5.45(12.22)$ \\
\hline \multicolumn{3}{|l|}{ Number of inhalations per attack day } \\
\hline Median (interquartile range) & $5.5(4.0-7.0)$ & $6.0(4.0-8.0)$ \\
\hline Mean (SD) & $6.49(3.85)$ & $6.49(3.13)$ \\
\hline \multicolumn{3}{|l|}{ Number of extra inhalations above baseline per attack day } \\
\hline Median (interquartile range) & $3.6(2.8-5.0)$ & $4.0(3.2-5.4)$ \\
\hline Mean (SD) & $4.21(1.97)$ & $4.66(2.28)$ \\
\hline Symptomatic worsening $+>4$ inhalations/day of rescue medication use above baseline & $n=33$ events & $\mathrm{n}=7 \mathbf{I}$ events \\
\hline \multicolumn{3}{|l|}{ Attack duration (days) } \\
\hline Median (interquartile range) & $2(2-5)$ & $2(2-6)$ \\
\hline Mean (SD) & $5.30(6.15)$ & $6.66(18.16)$ \\
\hline \multicolumn{3}{|l|}{ Number of inhalations per attack day } \\
\hline Median (interquartile range) & $8.4(6.5-11.0)$ & $8.4(7.00-11.00)$ \\
\hline Mean (SD) & $9.72(4.55)$ & $9.29(3.23)$ \\
\hline \multicolumn{3}{|l|}{ Number of extra inhalations above baseline per attack day } \\
\hline Median (interquartile range) & $6.5(5.0-7.9)$ & $5.9(5.2-8.0)$ \\
\hline Mean (SD) & $6.63(1.94)$ & $6.85(2.4 I)$ \\
\hline
\end{tabular}

Notes: Baseline rescue medication use was defined as the mean of the values recorded morning and evening during the last 10 days prior to randomization (when all patients were receiving budesonide/formoterol). This required $\geq 13$ valid values, including the evening assessment performed 10 days prior to randomization and the morning assessment performed on the day of randomization.

Abbreviations: BUD, budesonide; FORM, formoterol; SD, standard deviation.

\section{Oral Steroid Use}

Mean systemic corticosteroid use due to moderate/severe exacerbations over the treatment period at the $>2$ inhalations/day threshold was 6.56 days per patient-year for patients with no COPD symptom-related attacks, and 9.96 days per patient-year for those with $\geq 1$ attack. Mean systemic corticosteroid use at the $>4$ inhalations/day threshold was 6.80 days per patient-year for patients with no COPD symptom-related attacks, and 11.43 days per patient-year for patients with $\geq 1$ attack.

\section{Impact of Budesonide/Formoterol versus Formoterol Alone on Prevention of COPD Symptom-Related Attacks}

COPD symptom-related attacks occurred less frequently with budesonide/formoterol versus formoterol, with respective annualized rates of 0.27 and 0.49 attacks/ patient-year at the $>2$ inhalations/day threshold (Figure $4)$. The estimated rate reduction was $44 \%$ with budesonide/formoterol versus formoterol alone (rate ratio [RR]
0.56 ; $95 \%$ CI: $0.41,0.77 ; \mathrm{p}<0.001)$. At the $>4$ inhalations/day threshold, COPD symptom-related attacks also occurred less frequently with budesonide/formoterol versus formoterol, with annualized rates of 0.05 and 0.12 / patient-year, respectively (Figure 4). The estimated rate reduction was $57 \%$ with budesonide/formoterol versus formoterol alone (RR 0.43; 95\% CI: 0.26, 0.70; $\mathrm{p}<0.001)$.

Although these COPD symptom-related attacks were less frequent for budesonide/formoterol versus formoterol at the $>2$ and $>4$ inhalations/day thresholds, once a COPD symptom-related attack occurred, the characteristics were very similar in terms of duration and use of rescue medication between treatment groups (Table 2).

Mean baseline rescue medication use was 1.52 inhalations/day in the budesonide/formoterol treatment group, and 1.46 for formoterol alone. Rescue inhalation use per event day during COPD symptom-related attacks were 6.62 and 7.49 inhalations for the budesonide/formoterol and formoterol treatment groups, respectively, at the $>2$ 

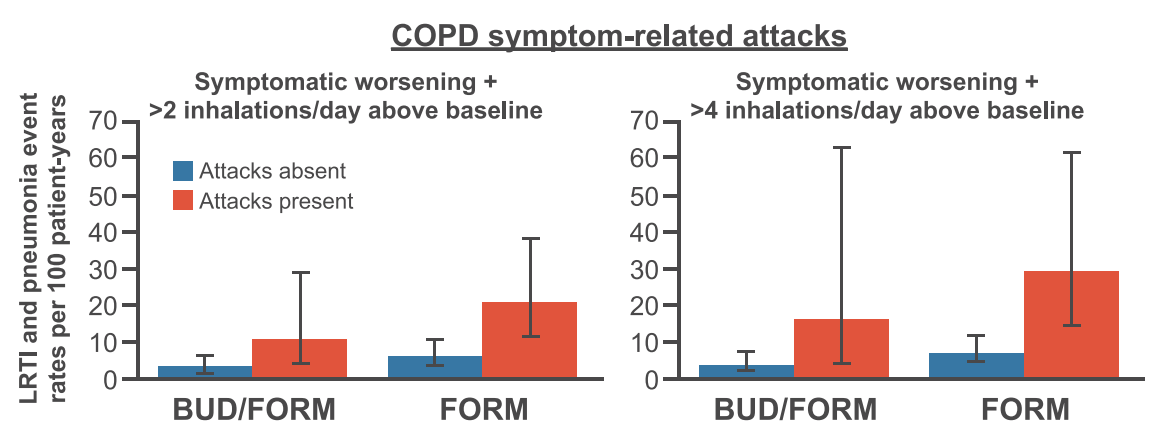

Moderate/severe exacerbations

Figure 5 Lower respiratory tract infections and pneumonia event rates per 100 patient-years.

Note: Error bars are $95 \% \mathrm{Cl}$.

Abbreviations: BUD, budesonide; FORM, formoterol; LRTI, lower respiratory tract infection.

inhalations/day threshold. At the $>4$ inhalations/day threshold, rescue medication use was 10.28 and 10.25 inhalations/event day for budesonide/formoterol and formoterol alone, respectively.

The mean event duration was 4.88 days and 5.45 days for budesonide/formoterol and formoterol alone, respectively, at the $>2$ inhalations/day threshold. At the $>4$ inhalations/day threshold, mean duration was 5.30 days for budesonide/formoterol and 6.66 days for formoterol alone.

In total, $13 \%$ of evaluable patients in the budesonide/ formoterol treatment group and $19 \%$ of patients in the formoterol treatment group had COPD symptom-related attacks at the $>2$ inhalations/day threshold. At the $>4$ inhalations/day threshold, $4 \%$ and $9 \%$ of budesonide/formoterol and formoterol patients had COPD symptomrelated attacks, respectively.

\section{LRTI and Pneumonia}

Rates (per 100 patient-years) of combined LRTI and pneumonia were lower for patients with no COPD symptomrelated attacks compared with $\geq 1$ attacks. These events also occurred at a lower rate with budesonide/formoterol compared with formoterol at both rescue medication use thresholds (Table S2 and Figure 5).

\section{Discussion}

This post hoc analysis of the RISE study showed that COPD symptom-related attacks, whether defined as $>2$ or $>4$ SABA inhalations per day above baseline in conjunction with $\geq 2$ consecutive days of worsening Anthonisen symptoms, are common and contribute to negative outcomes typically associated with moderate and severe exacerbation events.
These "COPD symptom-related attacks" would be classified by the GOLD report as "mild" COPD exacerbations. ${ }^{1}$ This is the first study to provide a quantifiable definition that might be easily used in clinical practice and research, and identifies meaningful outcomes related to these events. Indeed, our analysis has shown that such events may account for considerable morbidity and impaired quality of life beyond what could be regarded as "mild". We have shown that, irrespective of treatment with an ICS/LABA or LABA alone, COPD symptom-related attacks are associated with an increased risk of subsequent moderate/severe exacerbations and a clinically meaningful decline in HRQoL (SGRQ) and lung function $\left(\mathrm{FEV}_{1}\right)$. The patients included in the RISE study were on stable COPD maintenance therapy and were not exacerbating at study entry, reflecting a baseline rescue medication use for a COPD population meeting stability and eligibility criteria to enter a clinical trial, which may differ from rescue use in a more general COPD population. Consequently, these results are applicable to those patients not having frequent exacerbations and who are not overusing their rescue medication, as opposed to patients who are using multiple rescue inhalations a day. It was not clear which of the $>2$ or $>4$ inhalations/day above baseline thresholds provided the more discriminating definition, which may warrant further study. However, even at the lower threshold, significant morbidity occurred.

At both thresholds, the actual mean rescue medication use was considerably higher than the cut-offs we applied. Moderate/severe exacerbations were associated with lower mean rescue medication use, probably reflecting their early treatment with systemic corticosteroids and/or use of different exacerbation definitions. Experiencing a COPD symptom-related attack increased the likelihood of a subsequent moderate/severe exacerbation, but there was 
also a cumulative increase in risk with more attacks, showing that COPD symptom-related attacks are morbid events which should be reported and prevented.

Further evidence for the importance of worsening symptoms was that mean changes from baseline in SGRQ total scores for COPD symptom-related attacks were consistent with those seen with moderate/severe exacerbations. There was a lesser decrease in $\mathrm{FEV}_{1}$ with moderate/severe exacerbations versus COPD symptomrelated attacks, which likely reflects the treatment of moderate/severe exacerbations with systemic corticosteroids. Lung function was measured approximately 3 weeks after the start of an event, suggesting that lung function decrements persist for a substantial amount of time postevent, unless treated with systemic corticosteroids and/or antibiotics.

In addition to highlighting the incidence and importance of COPD symptom-related attacks, our analysis also showed that adding budesonide to formoterol significantly reduces the frequency of these attacks versus formoterol alone, without the increased risk of LRTI or pneumonia. ${ }^{14}$ As COPD symptom-related attacks led to increased risk of moderate/severe exacerbations, patients with more moderate-to-severe exacerbations would be subject to higher systemic corticosteroid exposure, which could increase or prolong immunosuppression, thus increasing the risk of infection.

As part of the study design, all patients received budesonide/formoterol during the run-in period, which was intended to standardize pre-randomization COPD maintenance treatment. Notable differences in event rates were observed when ICS was withdrawn in the formoterol treatment group, suggesting that ICS withdrawal carries risk for worsening symptoms across the spectrum of exacerbation severity.

Our analysis showed that COPD patients do use SABA routinely, albeit usually at a low baseline level, and that elevations in SABA rescue use associated with COPD symptom-related attacks are clinically meaningful and above normal day-to-day variability. Patients experiencing a "mild" attack with $\geq 2$ days of worsening symptoms and as little as $>2$ inhalations/day of SABA reliever above baseline had a markedly increased risk of future adverse health outcomes. Systemic corticosteroid exposure is also increased in the presence of COPD symptom-related attacks, and patients experiencing these attacks suffer prolonged reductions in lung function and prolonged increases in overall rescue medication use. Thus, the symptom burden is higher due to lack of prompt and more aggressive treatment with antibiotics and corticosteroids.

The quantification of COPD symptom-related attacks and their impact on patient health status, as well as their relationship to future risk of moderate/severe exacerbations, could make them a vital tool in clinical management of patients with COPD. Tracking daily reliever inhaler usage could help ensure that COPD symptom-related attacks do not go unreported, and additional treatment, when appropriate, can be initiated. Giving patients the ability to monitor for COPD symptom-related attacks could empower them to better manage their own condition, while health-care providers can use such measures to better evaluate their patients' therapy, improving health outcomes and reducing the risk of future moderate/severe exacerbations. A feasibility study of a digital COPD respiratory symptom tracker in a primary care setting found that patient compliance with daily symptom reporting was relatively high, although a relatively low number of patients initiated contact with their practitioner in response to alerts about elevated symptoms, confirming that these events are under-reported by patients. ${ }^{15}$ Whether tracking of extra rescue inhalations could better identify and enhance patient response remains unknown. If so, currently available smart inhalers could enable remote monitoring of digitally collected patient data to assess trends in reliever usage. ${ }^{16,17}$ Such a tool could alert healthcare providers that a future exacerbation is likely, allowing for even earlier intervention. A clear directive to patients to report COPD symptom-related attacks to their healthcare provider may enhance this process.

This study's strength is that it is the first to define and quantify COPD symptom-related attacks and to reveal their impact on patients with COPD. A further strength was that we used both symptoms and rescue medication use in our definition of these attacks, which established a high enough threshold to distinguish these attacks from day-to-day variability in COPD symptoms or rescue use unrelated to worsening symptoms.

A limitation of our analysis was that baseline rescue medication use and SGRQ and $\mathrm{FEV}_{1}$ measurements were assumed to reflect a stable baseline throughout the study. It could be argued that patients do not return to baseline after an exacerbation; EXACT, for example, resets the baseline following an exacerbation. ${ }^{9}$ Although an intrinsic part of this analysis, further study could overcome this limitation, for instance through the use of connected technology to get a clearer idea of baseline. The findings of this post hoc 
analysis should also be confirmed in a larger prospective study.

\section{Conclusion}

In conclusion, COPD symptom-related attacks are common and are associated with a significant impact on health outcomes. The findings from this post hoc analysis suggest that detection and recording of exacerbations not treated with systemic corticosteroids/antibiotics should be used in the clinical management of COPD, and should be accounted for as part of a more complete definition of COPD exacerbations moving forward. Budesonide/formoterol significantly reduces the frequency of these events versus formoterol alone, without an associated increase in the rate of LRTI and pneumonia.

\section{Abbreviations}

COPD, chronic obstructive pulmonary disease; HRQoL, health-related quality of life; SABA, short-acting $\beta_{2^{-}}$ agonist; SGRQ, St George's Respiratory Questionnaire; $\mathrm{FEV}_{1}$, forced expiratory volume in 1 second; HCRU, health-care resource utilization; OCS, oral corticosteroids;

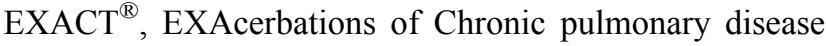
Tool; GOLD, Global Initiative for Chronic Obstructive Lung Disease; pMDI, pressurized metered dose inhaler; BID, twice daily; DPI, dry powder inhaler; ICS, inhaled corticosteroid; LABA, long acting $\beta_{2}$ agonist; LRTI, lower respiratory tract infections; $\mathrm{AE}$, adverse event; SAE, serious adverse event.

\section{Data Sharing Statement}

Data underlying the findings described in this manuscript may be obtained in accordance with AstraZeneca's data sharing policy described at https://astrazenecagrouptrials. pharmacm.com/ST/Submission/Disclosure. The corresponding author had full access to all the data and had final responsibility to submit for publication.

\section{Acknowledgments}

This study was supported by AstraZeneca. The authors would like to thank Stefan Courtney and David Candlish of inScience Communications, Springer Healthcare Ltd, UK, for providing medical writing support, which was funded by AstraZeneca in accordance with Good Publication Practice (GPP3) guidelines (http://www. ismpp.org/gpp3).

\section{Author Contributions}

All authors made substantial contributions to conception and design, acquisition of data, or analysis and interpretation of data; took part in drafting the article or revising it critically for important intellectual content; agreed to submit to the current journal; gave final approval of the version to be published; and agree to be accountable for all aspects of the work.

\section{Funding}

AstraZeneca funded the study and had a role in study design, data collection, data analysis, data interpretation, and writing of the report.

\section{Disclosure}

GTF reports grants, personal fees and non-financial support from AstraZeneca as part of the study. He has also received grants, personal fees and non-financial support from Boehringer Ingelheim, Novartis, AstraZeneca/Pearl Therapeutics, GlaxoSmithKline and Sunovion; grants and personal fees from Verona, Theravance and Sanofi; grants from Altavant; and personal fees from Innoviva, Mylan and Circassia outside the submitted work. FT, IG, LN, RL and TS are employees and shareholders of AstraZeneca. RL has patents EP2100598A1, EP2100599A1, and EP2666465 issued. AA and DL are employees of Phastar, which received funding from AstraZeneca to complete the statistical analyses. The authors report no other conflicts of interest in this work.

\section{References}

1. Global Initiative for Chronic Obstructive Lung Disease. Global strategy for prevention, diagnosis and management of COPD; 2020. Available from: https://goldcopd.org/. Accessed June 11, 2020.

2. Hurst JR, Vestbo J, Anzueto A, et al. Susceptibility to exacerbation in chronic obstructive pulmonary disease. $N$ Engl J Med. 2010;363 (12):1128-1138. doi:10.1056/NEJMoa0909883

3. Pavord ID, Jones PW, Burgel PR, Rabe KF. Exacerbations of COPD Int $J$ COPD. 2016;11:21-30.

4. Jinjuvadia C, Jinjuvadia R, Mandapakala C, Durairajan $N$, Liangpunsakul S, Soubani AO. Trends in outcomes, financial burden, and mortality for acute exacerbation of chronic obstructive pulmonary disease (COPD) in the United States from 2002 to 2010. COPD. 2017;14(1):72-79. doi:10.1080/15412555.2016.1199669

5. Guarascio AJ, Ray SM, Finch CK, Self TH. The clinical and economic burden of chronic obstructive pulmonary disease in the USA. Clinicoecon Outcomes Res. 2013;5:235-245.

6. Sato M, Chubachi S, Sasaki M, et al. Impact of mild exacerbation on COPD symptoms in a Japanese cohort. Int J COPD. 2016;11:1269-1278.

7. Vijayasaratha K, Stockley RA. Reported and unreported exacerbations of COPD: analysis by diary cards. Chest. 2008;133(1):34-41. doi:10.1378/chest.07-1692

8. Seemungal TA, Donaldson GC, Bhowmik A, Jeffries DJ, Wedzicha JA. Time course and recovery of exacerbations in patients with chronic obstructive pulmonary disease. Am J Respir Crit Care Med. 2000;161(5):1608-1613. doi:10.1164/ajrccm.161.5.9908022 
9. Jones PW, Lamarca R, Chuecos F, et al. Characterisation and impact of reported and unreported exacerbations: results from ATTAIN. Eur Respir J. 2014;44(5):1156-1165. doi:10.1183/09031936.00038814

10. Jones PW, Watz H, Wouters EFM, Cazzola M. COPD: the patient perspective. Int $J$ COPD. 2016;11(Special Issue 1st World Lung Disease Summit):13-20.

11. Wilkinson TM, Donaldson GC, Hurst JR, Seemungal TA, Wedzicha JA. Early therapy improves outcomes of exacerbations of chronic obstructive pulmonary disease. Am J Respir Crit Care Med. 2004;169(12):1298-1303. doi:10.1164/rccm.200310-1443OC

12. Ferguson GT, Tashkin DP, Skarby T, et al. Effect of budesonide/ formoterol pressurized metered-dose inhaler on exacerbations versus formoterol in chronic obstructive pulmonary disease: the 6-month, randomized RISE (Revealing the Impact of Symbicort in reducing Exacerbations in COPD) study. Respir Med. 2017;132:31-41. doi:10.1016/j.rmed.2017.09.002

13. Anthonisen NR, Manfreda J, Warren CP, Hershfield ES, Harding GK, Nelson NA. Antibiotic therapy in exacerbations of chronic obstructive pulmonary disease. Ann Intern Med. 1987;106(2):196-204. doi:10.7326/0003-4819-106-2-196
14. Janson C, Larsson K, Lisspers KH, et al. Pneumonia and pneumonia related mortality in patients with COPD treated with fixed combinations of inhaled corticosteroid and long acting $\beta 2$ agonist: observational matched cohort study (PATHOS). BMJ. 2013;346:f3306.

15. Criner GJ, Cole T, Hahn KA, Kastango K, Eudicone JM, Gilbert I. A feasibility study to examine real-world use of a daily digital chronic obstructive pulmonary disease (COPD) respiratory symptom tracker in a primary care setting. Presented at: American Thoracic Society International Conference; 2019; Dallas, TX, USA.

16. Sumino K, Locke ER, Magzamen S, et al. Use of a remote inhaler monitoring device to measure change in inhaler use with chronic obstructive pulmonary disease exacerbations. J Aerosol Med Pulm Drug Deliv. 2018;31(3):191-198. doi:10.1089/jamp.2017.1383

17. Blakey JD, Bender BG, Dima AL, Weinman J, Safioti G, Costello RW. Digital technologies and adherence in respiratory diseases: the road ahead. Eur Respir J. 2018;52(5):1801147. doi:10.1183/13993003.01147-2018

\section{Publish your work in this journal}

The International Journal of COPD is an international, peer-reviewed journal of therapeutics and pharmacology focusing on concise rapid reporting of clinical studies and reviews in COPD. Special focus is given to the pathophysiological processes underlying the disease, intervention programs, patient focused education, and self management protocols. This journal is indexed on PubMed Central, MedLine and CAS. The manuscript management system is completely online and includes a very quick and fair peer-review system, which is all easy to use. Visit http://www.dovepress.com/testimonials.php to read real quotes from published authors. 